\title{
FAINT IRAS NUMBER COUNTS AND GALAXY EVOLUTION
}

\author{
Carol J. Lonsdale \\ IPAC 100-22 \\ California Institute of Technology and Jet Propulsion Laboratory \\ Pasadena CA 91125
}

We present results of number count studies of IRAS deep Pointed Observations and the new Faint Source Survey. Since number counts are dominated by the population near the knee of the luminosity function, the results refer to starburst and active galaxies with typical luminosities $L_{60} \sim 10^{10.5-11} L_{\odot}$ and $L_{i r} / L_{b} \sim 3$, and not to 'normal' spirals or the 'ultraluminous' IR galaxies.

In addition to evolutionary phenomena, number counts will be affected by large scale structure variations. Therefore, in contrast to the approach of Franceschini et al. (1988), we have used a very simple evolutionary model (Hacking, Condon and Houck 1987; $\mathrm{HCH}$ ), to study the structure and to assess the first order ability of the IRAS data to detect luminosity evolution. This model is based on faint observed radio source counts (Condon 1984), and roughly corresponds to the rate of evolution that is capable of explaining the Butcher-Oemler effect. The entire luminosity function is assumed to be evolving in luminosity at a rate of $30 \% / \mathrm{Gyr}$, and color evolution is assumed to be negligible. This model is illustrated as the upper curve in the normalized differential $60 \mu \mathrm{m}$ number count diagram below. The curve is marked at intervals by the expected median redshift. The lower curve represents the counts expected in the case of no evolution. The fall off of this curve from a straight horizontal line is entirely due to the ' $\mathrm{K}$-correction'.

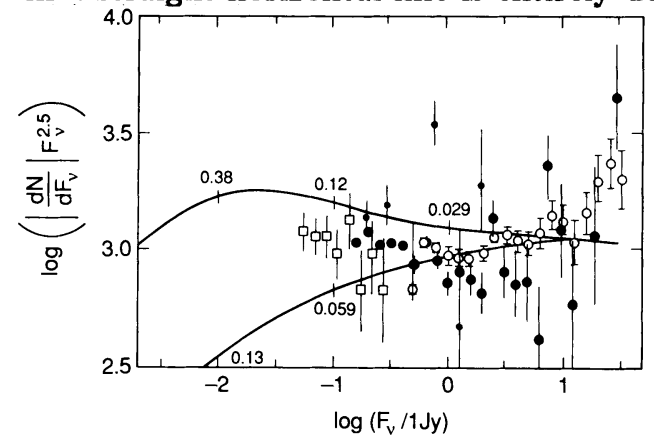

Figure 1. Normalized differential $60 \mu m$ source counts $\left(J y^{2.5} s r^{-1}\right)$.

416

D. E. Osterbrock and J. S. Miller (eds.), Active Galactic Nuclei, 416-417.

(c) 1989 by the IAU. 
In the figure are illustrated $60 \mu m$ normalized differential number counts for various IRAS samples. The error bars represent Poisson statistics. The open circles are Point Source Catalog counts for $\mathrm{b}^{I I}>50^{\circ}$ and the open squares are ultradeep counts in a small field at the ecliptic pole $(\mathrm{HCH})$. The small filled circles are deep Pointed Observation counts in 22 small southern fields (Lonsdale and Hacking 1988; LH), and the larger filled circles (Lonsdale, Hacking and Conrow 1988; LHC) are new counts from a preliminary sample selected from the Faint Source Survey (FSS).

The faint Pointed Observation number counts are significantly higher than predicted by the non-evolving model. The new Faint Source Survey database does not reach as deep as the Pointed Observations, but contains far more sources so that the number count statistics are much superior. The FSS sample displayed in the figure covers only a fraction of the extragalactic sky, yet already the error bars on the deepest counts are negligible. The FSS counts confirm the faint count excess over the non-evolving model.

At present the interpretation of the faint $60 \mu m$ number count excess is restricted by the dispersion in the bright counts, which is due to local large scale structure (see, eg., LH). The models have been normalized by $\mathrm{HCH}$ to the Point Source Catalog counts at $1 \mathrm{Jy}$; however it is not yet clear that this is a good representation of the local ambient density. Cirrus contamination must also be eliminated. Lonsdale, Hacking and Conrow (1988) are addressing these issues using the entire Faint Source Survey data base.

If the models are presently correctly normalized then the faint number count excess over the non-evolving model is indicating either the existence of evolution, or of a structure of size $>300(\mathrm{H} / 50)$ Mpc. The latter possibility seems unlikely since the excess is seen in six different and widely spaced fields in the FSS sample of LHC. Redshift data is being collected for a subset of about 2000 galaxies to study this issue.

Evolution of the $60 \mu \mathrm{m}$ number counts would most simply imply that the $60 \mu \mathrm{m}$ population is evolving in one or more of (a) the overall rate of star formation and/or non-thermal activity of each galaxy, at about $20 \% / \mathrm{Gyr}$; (b) the percentage of galaxies experiencing such star formation and/or activity; or (c) the timescale for a typical starburst or active period.

Condon, J.J. 1984, Ap. J., 287, 461.

Franceschini, A., Danese, L., De Zotti, G., and Xu, C. 1988, M.N.R.A.S., 233, 175.

Hacking, P.B., Condon, J.J., and Houck, J.R., 1987, Ap. J. Letters, 316, L15 ( $\mathrm{HCH})$.

Lonsdale, C.J., and Hacking, P.B. 1988 Ap. J. in press, (LH).

Lonsdale, C.J., Hacking, P.B., and Conrow, T.P. 1988, in preparation, (LHC). 